\title{
OVERVIEW OF THE MARS LASER COMMUNICATIONS DEMONSTRATON PROJECT
}

\author{
Bernard L. Edwards ${ }^{1}$, Stephen A. Townes ${ }^{2}$, Roy S. Bondurant ${ }^{3}$, Joseph J. Scozzafava ${ }^{3}$, Don M. Boroson ${ }^{3}$, \\ Ben A. Parvin ${ }^{2}$, Abhijit Biswas ${ }^{2}$, Alan D. Pillsbury ${ }^{3}$, Farzana I. Khatri ${ }^{3}$, Jamie W. Burnside ${ }^{3}$, David R. Bold ${ }^{3}$, \\ Daniel V. Murphy ${ }^{3}$, Alexander K. McIntosh ${ }^{3}$, David O. Caplan ${ }^{3}$, Alan E. DeCew ${ }^{3}$, Jayant Sharma ${ }^{3}$, \\ Richard J. Fitzgerald ${ }^{1}$, Samuel H. Zingales ${ }^{2}$, and Ramon DePaula ${ }^{4}$ \\ ${ }^{1}$ NASA Goddard Space Flight Center, Greenbelt, MD 20771, USA \\ ${ }^{2}$ Jet Propulsion Laboratory, Pasadena, CA 91109, USA \\ ${ }^{3}$ MIT Lincoln Laboratory, Lexington, MA 02420, USA \\ ${ }^{4}$ NASA Headquarters, Washington, DC 20546, USA \\ E-mail address: Bernard.L.Edwards@nasa.gov
}

\begin{abstract}
This paper provides an overview of the Mars Laser Communications Demonstration Project, a joint project between NASA's Goddard Space Flight Center (GSFC), the Jet Propulsion Laboratory, California Institute of Technology (JPL), and the Massachusetts Institute of Technology Lincoln Laboratory (MIT/LL). It reviews the strawman designs for the flight and ground segments, the critical technologies required, and the concept of operations. It reports preliminary conclusions from the Mars Lasercom Study conducted at MIT/LL and on additional work done at JPL and GSFC. The lasercom flight terminal will be flown on the Mars Telecom Orbiter (MTO) to be launched by NASA in 2009, and will demonstrate a technology which has the potential of vastly improving NASA's ability to communicate throughout the solar system.
\end{abstract}

\section{INTRODUCTION}

In the near future the National Aeronautics and Space Administration anticipates a significant increase in demand for long-haul communications services from deep space to Earth. Distances will range from 0.1 to $40 \mathrm{AU}$, with data rate requirements in the 1's to 1000's of Mbits/second. The near term demand is driven by NASA's Space Science Enterprise which wishes to deploy more capable instruments onboard spacecraft and increase the number of deep space missions. The long term demand is driven by missions with extreme communications challenges such as very high data rates from the outer planets, supporting sub-surface exploration, or supporting NASA's Human Exploration and Development of Space Enterprise beyond Earth orbit.
NASA's Goddard Space Flight Center, the Jet Propulsion Laboratory, and MIT's Lincoln Laboratory are working together to demonstrate optical communications on the 2009 Mars Telecom Orbiter (MTO). The Mars Laser Communications Demonstration Project will demonstrate one possible solution in meeting NASA's future long-haul communication needs. Lasercom sends information using beams of light and optical elements, such as telescopes and optical amplifiers, rather than RF signals, amplifiers, and antennas. Near-Earth lasercom systems have been demonstrated (GeoLITE and GOLD in the U.S. and SILEX in Europe), and the technology has the potential to revolutionize deep space communications.

NASA sponsored the Mars Lasercom Study at MIT/LL this past year to develop the demonstration concept and some strawman designs. The demonstration Mars terminal is being designed to provide a continuous data link of between 1 and 100 Mbits/second from Mars to Earth, depending on the instantaneous distance and atmospheric conditions. The $100 \mathrm{Mbits} / \mathrm{second}$ data rate will be a significant performance increase over today's RF systems. The project is planning to use ground terminals capable of receiving the encoded laser beam and transmitting an uplink beacon laser to the flight terminal, for active tracking and pointing control of the narrow laser beam. Critical technologies for receiving the deep space signal include low-cost large collection apertures and low-noise photoncounting detectors. The project is investigating using existing astronomical telescopes as well as building one high performing terminal to allow operations close to the Sun.

Lasercom will enable bandwidth-hungry instruments, such as hyper-spectral imagers, synthetic 
aperture radar (SAR) and instruments with high definition in spectral, spatial or temporal modes to be used in deep space exploration. The Mars Laser Communications Demonstration Project will provide much needed engineering insight by the end of this decade.

\section{OVERVIEW OF DEEP SPACE LASERCOM}

Several organizations in the United States, Europe, and Japan are working on multi-Gbits/second lasercom systems for near Earth applications. Unfortunately, the technology suitable for near Earth does not easily extend to deep space requirements. Figure 1 shows inter-planetary link requirements compared to lasercom systems in geosynchronous orbit at Earth.

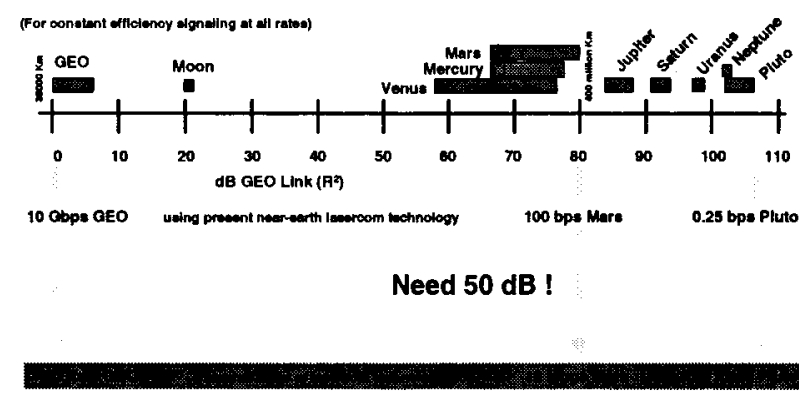

Figure 1: Deep Space Lasercom Compared to GEO Systems

As shown in the figure, Mars is as much as almost $80 \mathrm{~dB}$ farther out than GEO. Simply transporting a $10 \mathrm{Gbits} / \mathrm{second}$ lasercom system designed for near Earth applications from Earth to Mars would result in only 100 bits/second. An improvement of $50 \mathrm{~dB}$ is required to provide $10 \mathrm{Mbits} / \mathrm{second}$ from Mars.

Doing a few simple things like increasing the transmit aperture and laser power can provide a few dBs. A significant number of the required dBs can be obtained by increasing the receive aperture size from 10's of centimeters to between 5 and 10 meters. This is analogous to what is done by today's RF based Deep Space Network. The last dBs will have to come through the use of more efficient signaling, detection architectures, and high performance error-correcting codes that operate $0.5-0.75$ $\mathrm{dB}$ from capacity.

The fiber telecommunications industry uses optical pre-amplified receivers with simple modulation formats such as on-off keying (OOK). They have also used pre-amplified Differential Phase Shift Keying (DPSK) for very high-speed free space links. Although fairly efficient, especially with near capacity achieving forward error correction (FEC) coding, neither of these formats achieves anywhere near the ultimate efficiency of coded optical communication. They are used, though, because as of today, they are the best techniques for achieving the high gain-bandwidth performance required in efficient receivers at multi-Gbits/second rates. Highorder alphabet size modulations, such as low dutycycle pulsed formats, are known to provide high efficiency at the cost of considerable bandwidth expansion. For the deep space optical channel, this extra bandwidth is well within technological limits, and low-noise photon-counting reception is the most efficient means known to date for receiving it. Photon-counting detectors have been developed that detect photons with a time resolution set by an external clock.

In low-noise photon-counting receivers, spatial resolution is not required so "photon buckets", or large low cost optical collectors, are feasible. Unfortunately, background light degrades the efficiency of pulsed systems and large apertures lead to a lot of background light. This can be overcome, however, by using an even larger aperture to gather more signal photons; signal photons will be gathered faster than the background photons. Getting above the atmosphere, or at least above the turbulence, would greatly decrease the amount of background seen by the receiver. The Mars Lasercom Study showed that putting a receiver in space would reduce the aperture size by approximately $10 \mathrm{~dB}$ compared to a ground based receiver.

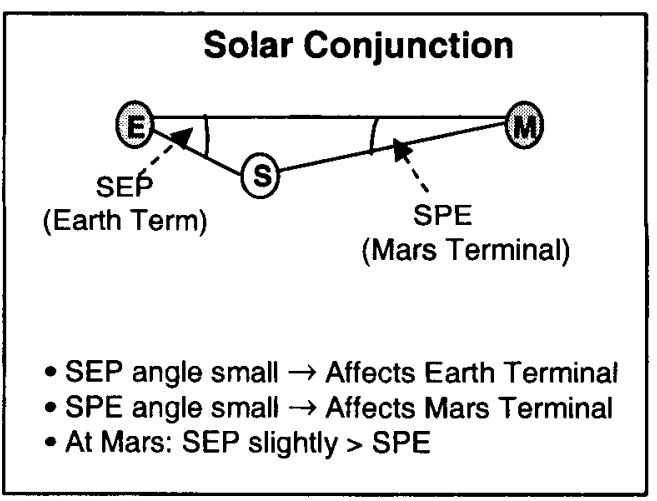

Figure 2: The Affect of Solar Conjunction

Another major area of concern for deep space lasercom is the need to work very close to the Sun. This is easily seen when considering the outer planets (e.g. from Pluto, the Earth is always very close to the Sun), but is also very important for a system at Mars. Figure 2 illustrates what is happening during solar conjunction. SEP is the Sun-Earth-Probe 
angle while SPE is the Sun-Probe-Earth angle. Small SEP angles interfere with the Earth terminal's ability to acquire and track the lasercom signal. Small SPE angles interfere with the Mars terminal's ability to acquire and track the uplink beacon laser from Earth. During solar opposition, small SPE angles also affect the Mars terminal as the Earth is again very close to the Sun.

Each day there is a time of sunrise, Mars rise, sunset, and Mars set. Most of the time, both the Sun and Mars will be in the sky simultaneously. From a lasercom systems engineering perspective, a critical design driver is the fact that Mars is simultaneously at its farthest distance and at its smallest SEP angle. The communication outages that arise when either the Earth or Mars is too close to the Sun have been evaluated for various SPE angles (and the corresponding SEP angles during solar conjunction) and are shown in table 1 .

\begin{tabular}{|l|l|l|}
\hline $\begin{array}{l}\text { SPE Angle } \\
\text { (Degrees) }\end{array}$ & $\begin{array}{l}\text { SEP Angle } \\
\text { (Degrees) }\end{array}$ & $\begin{array}{l}\text { Outage } \\
\text { (Days) }\end{array}$ \\
\hline 2 & 2.8 & 23 \\
\hline 4 & 5.7 & 49 \\
\hline 6 & 8.6 & 75 \\
\hline 8 & 11.4 & 100 \\
\hline 10 & 14.3 & 126 \\
\hline 15 & 21.9 & 190 \\
\hline 20 & 28 & 255 \\
\hline
\end{tabular}

Table 1: Annual Communication Outages in Days vs. SPE angles

The Mars Lasercom Study considered placing Earth terminals on the ground, in the air, and in space. Without considering weather, at least 3 terminals are needed for a ground based network for near $100 \%$ availability due to the rotation of the Earth. When random weather outages are taken into account, the number of terminals increase to about 9 depending on the weather and seeing conditions at the proposed sites. Balloon-borne terminals could number as few as 2 if the balloons allowed wide telescope viewing angles and if they could be placed near the poles, but a more realistic number is about six. Finally, 2 space terminals could suffice. The size of the aperture required at Earth is smallest in space where only the occasional Mars crossing adds background light. Balloon and earth terminals must be larger because of the scattered sunlight in the Earth's atmosphere and, in the case of ground terminals, turbulence which requires a large blur circle (i.e. many spatial modes of background light). More study and analysis needs to be conducted to determine the best course of action for NASA in the long term, but for the Mars demonstration in 2009, all Earth terminals will be located on the ground.

\section{THE MARS TERMINAL}

The primary functions of the Mars terminal are: to efficiently generate optical power that can have data modulated onto it; transmit this optical power through efficient optics; and aim the very narrow beam at earth, despite platform vibrations, motions, and distortions. The Mars terminal architecture pursued to meet these requirements includes: a transmitter consisting of a 5-Watt Ytterbium-doped fiber optical amplifier at $1.06 \mu \mathrm{m}$ in a MasterOscillator-Power Amplifier (MOPA) configuration; a $30 \mathrm{~cm}$ diffraction-limited telescope; 64-ary Pulse Position Modulation (PPM) with near-capacityachieving concatenated coding; and pointing and stabilization based on an uplink earth beacon with inertial stabilization. Each of these subsystems is described in the following sections.

\section{$\underline{\text { Transmitter }}$}

The Master Oscillator Power Amplifier (MOPA) transmitter architecture is modular, allowing for a more flexible choice of waveforms and independent design and optimization of the laser, modulator, and power amplifier. The design is commonly used for high rate optical communications in the telecom industry at Mbits/second rates to 10's of Gbits/second and beyond. The MOPA transmitter architecture selected for the strawman design is shown in Figure 3.

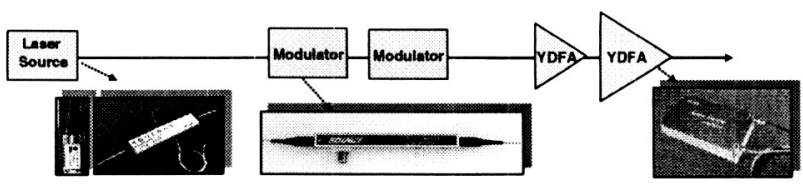

Figure 3: MOPA Transmitter Architecture

The master laser selected for the strawman design is a commercially available fiber distributed feedback (DFB) laser, which consists of a DFB fiber Bragg grating (FBG) written into Ytterbium doped fiber, pumped by a $980 \mathrm{~nm}$ laser diode. Both the pump laser and FBG are mature technologies, used throughout the telecom industry.

The modulator selected is a LiNO3 Mach-Zehnder modulator (MZM), a technology that is also used 
throughout the telecom industry (at $1.5 \mu \mathrm{m}$ ) for high rate, high performance communications. Commercially available MMZs were evaluated and the following parameters were observed: Insertion Loss < $3 \mathrm{~dB} ; 3 \mathrm{~dB}$ Bandwidth > $10 \mathrm{GHz} ; \mathrm{V} \pi$ (@1 GHz) 2 volts; ER @ $1 \mathrm{GHz} \sim 20 \mathrm{~dB}$. A cascade of two MMZs in series is used to provide $\sim 40 \mathrm{~dB}$ extinction ratio which is sufficient for generating large constellation M-PPM waveforms without penalty.

High power Ytterbium doped fiber amplifiers (YDFAs) are commercially available from several vendors with $\sim 15 \%$ wall plug efficiency. In the strawman design a filtered and isolated high gain preamplifier precedes the power amplifier, providing sufficient power to saturate the subsequent power amp and efficiently extract the $5 \mathrm{~W}$ average output.

\section{Modulation And Coding}

A key aspect in designing a Mars-Earth communications link with the highest power-efficiency is an effective modulation format with error-correction coding. The low-duty cycle modulation format selected for the Mars terminal is Pulse Position Modulation (PPM). In the PPM modulation format, exactly one out of $\mathrm{M}$ pulses is on, thus delivering $\log 2(\mathrm{M})$ bits for every pulse, as shown in Figure 4.
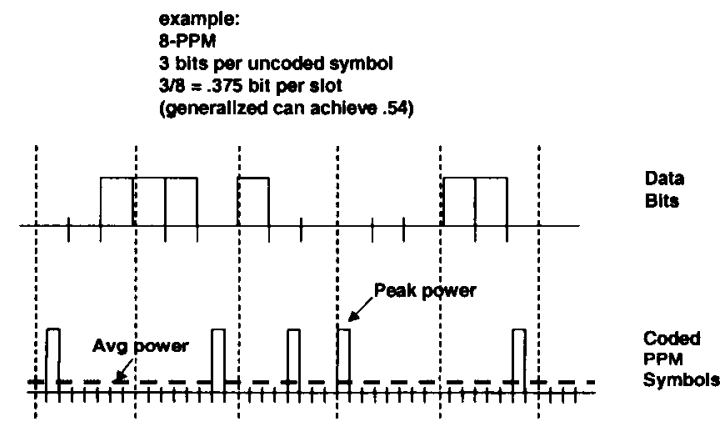

Figure 4: PPM Modulation Format Example.

The modulator is paired with a Forward Error Correction (FEC) encoder designed specifically to match the PPM channel. The most common FEC code paired with PPM has long been ReedSolomon (RS) codes since they both deal in blocks of $\log 2(\mathrm{M})$ bits. One advantage of RS codes is that off-the-shelf chipsets exist for coder/decoders. But, one disadvantage is that RS codes do not achieve capacity, even with a more complex soft-decision decoder. Another possibility is to use a Turbo-Code using iterative decoding which comes within $1 \mathrm{~dB}$ or so of capacity. Codes are typically constructed by two or more constituent part, separated by long interleavers, which implicitly create very long codewords. The decoder finds a constituent code, but outputting values akin to maximum likelihood information (called "extrinsic information."). This information is then de-interleaved and applied to the decoder for the other constituent part. The process is iterated 5-10 times and the resultant output bits are very nearly the maximum likelihood (ML) decoded values for the very long codewords, even though the true ML calculation would have been extremely difficult.

We can achieve within $\sim 1 \mathrm{~dB}$ of capacity using a pulsed modulation with a photon counting receiver. Operating curves are shown in Figure 5 for various alphabet size $\mathrm{M}$ with 1 noise photon per slot. For example, with 64-ary modulation, trying to achieve $10 \mathrm{Mb} / \mathrm{s}$ (or 0.01 bits per slot with a $1 \mathrm{~ns}$ pulse) requires about $-18 \mathrm{~dB}$ photons per ns slot.

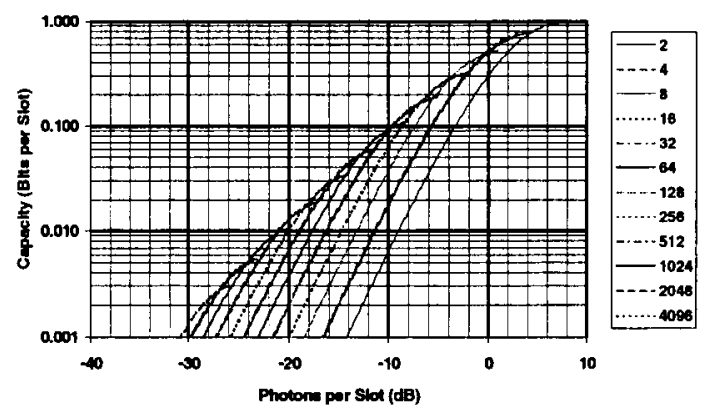

Figure 5: Pulsed Modulation, Photon-Counting Operating Curves for Various Alphabet Size M with Noise of 1 Count per Slot.

\section{Pointing And Stabilization System}

Figure 6 shows a block-diagram of the pointing control hardware for an inertial reference aided beacon-tracking terminal. The major components are:

- Miniature Inertial Reference Unit (MIRU)

- Fast steering mirror (FSM)

- Fast readout quad-cell

- Slow beacon camera and transmit monitor

- Point-ahead mirror (PAM)

The MIRU is an inertially stabilized platform for a collimated laser source. It is currently being developed by Applied Technology Associates of Albuquerque, NM under a Phase II SBIR for NASA. It is functionally similar to the Inertial Pseudo-Star Reference Unit (IPSRU) developed by Draper Laboratory for the Air Force Phillips Laboratory's

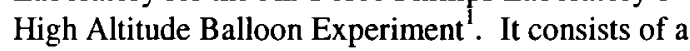


combination of magnetohydrodynamic inertial sensors, voice-coil actuators, proximity sensors, and a precision gyroscope. The quiescient jitter of the stabilized platform is expected to be less than 100 nrad. An extended corner cube folds the collimated laser light into the lasercom telescope.

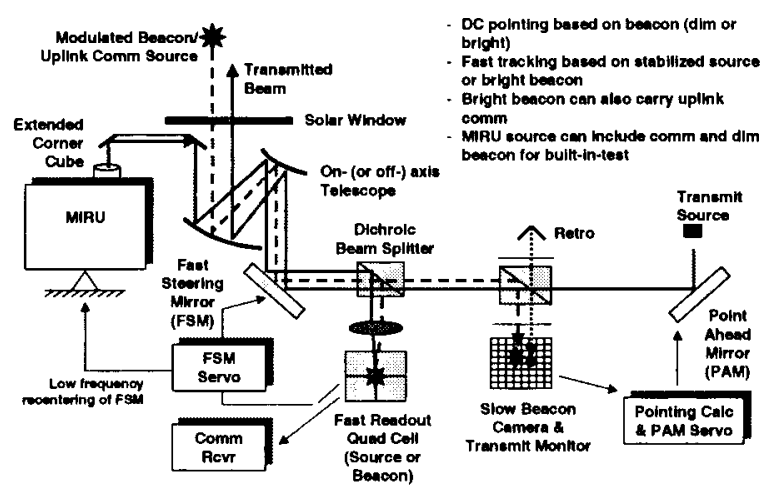

Figure 6: MIRU-Aided Beacon Tracking Diagram

The fast steering mirror is used to steer the incoming and outgoing beams within the telescope Field of Regard, and to stabilize the beams in order to reject spacecraft-borne motions.

The fast readout quad-cell is designed to detect modulated optical power and allow for closed-loop tracking of a modulated source via the FSM control loop servo. The fast readout quad cell can either detect a modulated local source on the MIRU stabilized platform (to stabilize the telescope relative to the local "pseudo-star"), or, if sufficient power is available, the fast readout quad-cell can directly sense the modulated uplink beacon and allow the FSM servo to track the uplink beacon. In general, the beacon uplink power may not be sufficient for high-bandwidth tracking, so the stabilized source on the MIRU provides a stable reference to allow closed loop tracking and rejection of spacecraft motions.

The slow beacon camera and transmit monitor provides two primary functions: it allows tracking of a dim beacon signal in order to correct for MIRU bias and drift; and it allows for tracking of the transmitter source in order to correctly align the receive and transmit beams. Orientation of the transmit beam about the line-of-sight is based on the spacecraft attitude determination and control system.

The point-ahead mirror is required when operating in direct beacon tracking mode (a mode to be demonstrated depending on available beacon power and atmospheric conditions). Since the fast steering mirror centers the tracked uplink beacon on the quad cell during this mode, an extra degree of freedom is needed to apply the point ahead angle to the transmit beam. Note that the point-ahead mirror is not required when using the MIRU for aided tracking and stabilization - the MIRU can provide the extra degree of freedom to offset the transmit and the receive beams.

\section{$\underline{\text { MIRU-Aided Pointing }}$}

During MIRU-aided pointing, the MIRU points a locally stabilized collimated source, nominally along the telescope boresight, but it can be offset by +- $2.5 \mathrm{mRad}$ to cover a large field of regard. When the FSM servo loop tracks this low-jitter source, the received light at the slow beacon camera and transmit monitor will be stabilized to the jitter level of the MIRU plus the jitter due to the FSM servo tracking performance. Thus, the light at the slow beacon camera and transmit monitor will be lowjitter, and the camera data may be used to detect a slow, weak uplink beacon (nominally $1 \mathrm{~Hz}$ tracking bandwidth). The position of the transmit beam relative to the receive beacon defines the point ahead angle, and is directly detected from the slow beacon camera and transmit monitor data. The MIRU pointing bias is then modified until the point-ahead angle (sensed at the slow beacon camera and transmit monitor) is the desired value. The desired point-ahead angle is calculated off-line based on the orbital geometry of the Mars and Earth terminals.

\section{$\underline{\text { Mars Terminal Configuration }}$}

The Mars lasercom terminal utilizes a $30.5 \mathrm{~cm}$ aperture $15 \mathrm{X}$ on-axis telescope. The $1 \mathrm{mrad}$ FOV required to accommodate the spacecraft pointing errors is readily accommodated without significant wavefront quality degradation.

A solar window is included to reject at least $80 \%$ of the solar load while passing more than $90 \%$ of the beacon and communications wavelengths. This greatly reduces the thermal loads on the telescope mirrors and metering structure and reduces the size of the thermal radiator. A shutter is provided to block the beam path in case some spacecraft anomaly causes the Sun to be directly on-axis; this will prevent damage to the tracking and transmitting optics.

A small portion of the transmit beam can be directed onto a $\mathrm{CCD}$ (the slow beacon and transmit 
monitor) by means of a retroreflector to boresight the transmit and beacon beam paths. Since the CCD can not distinguish between the two beams, only one beam can illuminate the detector at a time.

A Point Ahead Mirror (PAM) is provided to allow the beacon and transmit beam paths to be offset by the appropriate point ahead angle.

A block diagram of the Mars terminal configuration is shown as Figure 7.

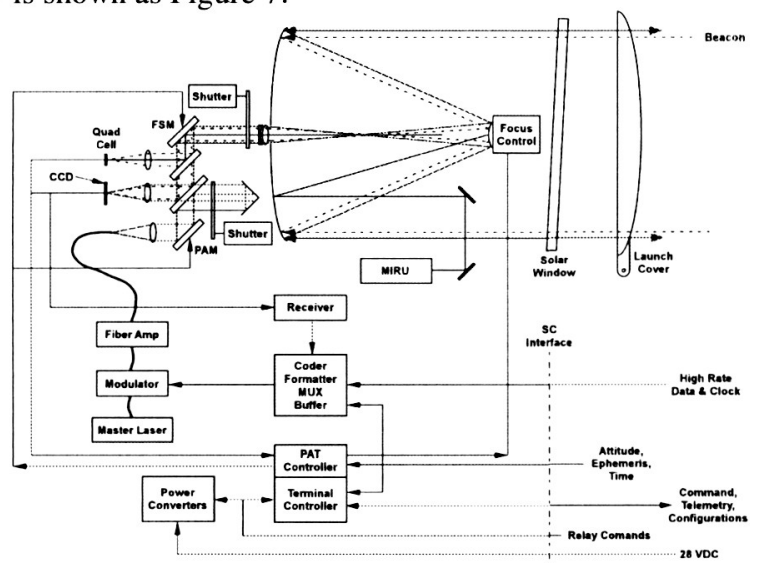

Figure 7: Terminal Configuration Block Diagram

\section{Telescope and Electronics}

The Mars terminal consists of separate optical and electronic modules mounted to the Mars Telecom Orbiter (MTO). The MTO will maintain Earth pointing to within $\pm 1 \mathrm{mrad}$, limiting the maximum sun anglesto $40^{\circ}$ off the spacecraft pointing direction. The configuration of the Mars terminal is shown as Figure 8.

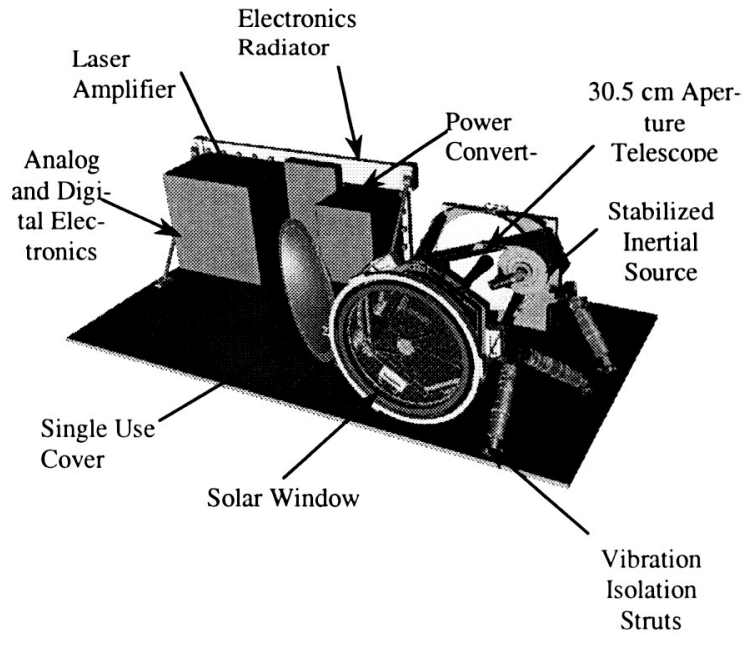

Figure 8: Mars Terminal Configuration
The Mars terminal will include a passive vibration isolation system. Lower resonant frequency systems provide better isolation of high frequency disturbances but require longer stroke struts than a higher frequency system. Low frequency isolation systems are more prone to interacting with the launch vehicle avionics during launch and so may need to be caged during launch. The proposed 20 $\mathrm{Hz}$ isolation system is a good compromise between the three constraints of stroke, isolation, and launch vehicle interactions. Such a system should not need to be caged during launch, particularly if it is a highly damped system. In this way, the isolation system also protects the optical components during launch.

The telescope consists of an Invar metering structure with Ultra Low Expansion (ULE) glass mirrors. The telescope is athermalized by having the baseplate made of aluminum. If the telescope warms up the metering structure would get slightly longer, tending to defocus the telescope. However, as the baseplate also warms up the splay of the Invar metering truss increases which tends to foreshorten the structure. The geometry of the metering structure was selected to balance these two effects.

Protective covers fabricated from molded carbon fiber material will be included to support the Multilayered Insulation (MLI) that will cover the telescope and the small optics on the rear of the telescope baseplate.

The electronics module is mounted to a thermal radiator which is thermally isolated from the spacecraft. This radiator will have heaters to maintain a minimum survival temperature of $-30^{\circ} \mathrm{C}$ and would be sized to limit the peak operating temperature to $50^{\circ} \mathrm{C}$ during times of peak power dissipation.

The telescope assembly includes a cover to protect the solar window up until the first use after launch. It also includes a thermal radiator to dissipate the solar load and internal dissipation from the FSM and tracking detector.

The rear end of the telescope assembly supports a small optical bench, shown in Figure 9, which contains the tracking, transmitting, and boresighting optics. The Fast Steering Mirror (FSM) is located at the telescope's exit pupil to minmize beam walk as the FSM corrects for spacecraft pointing errors. Both the FSM and the Point Ahead Mechanism (PAM) are based on commercially available mechanisms. The transmitter collimator is fed with a single-mode optical fiber with the laser located in 
the electronics module. The quadrant detector assembly includes preamplifiers to boost the signal level prior to sending the signals to the electronics module.

A shutter assembly is included to block solar illumination of the CCD detector, quadrant detector, and transmit fiber for those times when the sun is very close to being directly on-axis. No elements are required for boresighting the system.

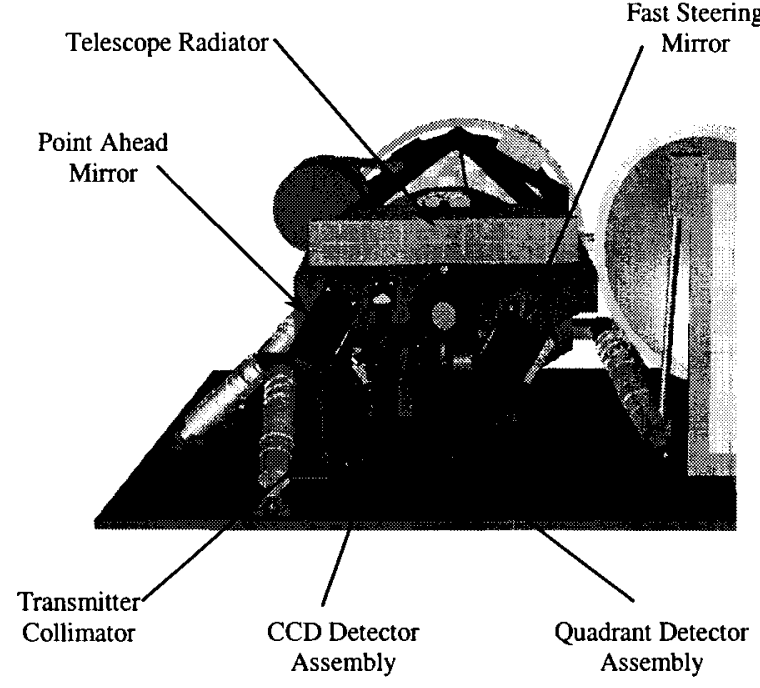

Figure 9: Small Optics Assembly

\section{THE EARTH TERMINAL}

The Earth terminal must provide two functions; receive the communications signal from Mars and transmit an uplink beacon beam so that the Mars terminal points to the correct location on the Earth.

The receiver must provide a collector large enough to capture adequate power to support the data rate; couple this light onto low noise, efficient detectors while trying to minimize the coupled background light; and perform synchronization, demodulation, and decoding of the received waveform.

The uplink beacon, transmitted from the vicinity of the receive terminal, must provide a pointing reference to establish the Mars terminal beam pointing direction. The requirement for such a beacon is that it be bright enough to support a $1-\mathrm{Hz}$ track by the Mars terminal to overcome low-frequency drift in the on-board inertial pointing reference.

The current plan is to have at least one ground terminal capable of operating with Sun-Earth-Probe angles as low as 3 degrees to minimize the outage around superior conjunction and with an equivalent aperture diameter of $5-10 \mathrm{~m}$. If affordable, multiple ground terminals will be deployed to demonstrate weather mitigation and other operations strategies.

\section{Lincoln Distributed Optical Receiver Architecture}

An array of small telescopes provides many benefits, when paired with low-noise (thermoelectrically-cooled) photon counters. Performance is very nearly the same as with a single, highperformance telescope, both in the bright, turbulent atmosphere and in the vacuum channel. (Different detector design optimizations are used in these two extremes.) The modular nature allows for many options of cost reduction, and is directly scalable to much larger (or smaller) total apertures with only a linear growth in cost. Perhaps one of the biggest benefits is the fact that small telescopes can include sunshades that would allow operation to within a very few degrees of the sun, thus keeping availability very high.

A large receiver can be efficiently constructed using multiple small telescopes, each with its own detectors. A master $1 \mathrm{GHz}$ (i.e., the slot rate) clock, slot-synchronized to the incoming stream, would be fed to the array. Each telescope is told its time offset as calculated throughout the day by a central processor, which also tells it where to point. Fine tracking can be done by each telescope by watching the detector array and fine pointing with the gimbal. All detections are sent via a $100 \mathrm{Mbps}$-class Ethernet star network to a fast switch which sends all the measurements to the decoding electronics.

Slot synch offsets are monitored via dithering techniques. PPM word and FEC codeword synch can be implemented in a number of ways including startup sequences, fixed deadtime patterns which add lines to the received spectrum (at the cost of increased peak power,) or by dedicating a small TDM subchannel to synch patterns. The receive array architecture is shown in Figure 10.

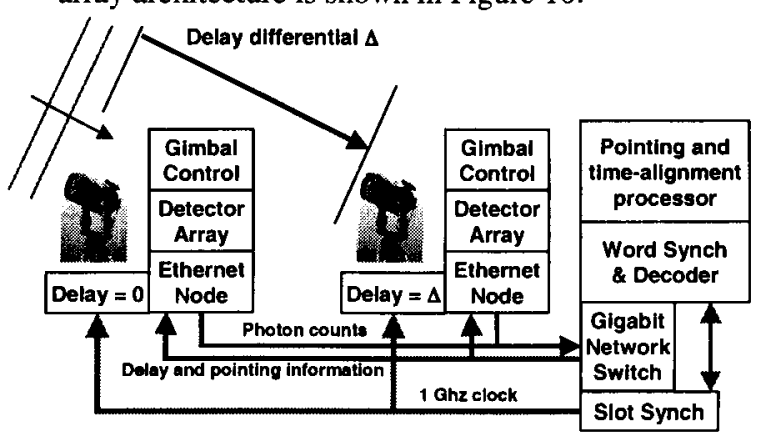

Figure 10: Receive Array Architecture 
The array architecture scales very well to spacebased systems. In either case, the selection of telescope size and number can be made to minimize cost while keeping performance the same. An extra $1 \mathrm{~dB}$ more receiver area costs, in general, $1 \mathrm{~dB}$ more in dollars.

\section{Sunshade}

In order to maintain system performance while operating within $3^{\circ}$ of the sun it is necessary to prevent the sun from illuminating the primary mirror. A sunshade that is $30 \mathrm{~cm}$ long with cells $1.5 \mathrm{~cm}$ wide and $10 \mathrm{~cm}$ high will meet this requirement. The use of an equatorial mount allows a relatively simple design consisting of flat panels. The panels are oriented perpendicular to the mount elevation axis and the $3^{\circ}$ sun angle. The internal surfaces of the sunshade would be coated to achieve very low reflectivity.

The large sunshade cells allow simple fabrication. We will investigate the possibility of extruding the cells in a process similar to the fabrication of commercial heat sinks. This is possible because with $0.64 \mathrm{~mm}$ thick cells the sunshade only reduces the telescope collection area by approximately $0.3 \mathrm{~dB}$.

When operating telescopes at night the outer optical element must be maintained at temperatures slightly above the dew point to prevent condensation. This will be done by adding low power heaters, approximately $15 \mathrm{~W}$, to the sunshade.

\section{Detectors}

One type of detector being considered for this project is an InGaAsP/InP Geiger-Mode APD. Geiger-Mode APDs (G-M APD) can be thought of as photon-to-digital converters which produce a digital logic-compatible voltage transition in response to a single incident photon. In this way G-M APDs completely eliminate many of the traditional sources of noise (read-noise, amplification noise, etc) involved in photon detection with analog receivers. In the G-M APD focal planes proposed for the Earth receiver system, each pixel is mated to a digital timing circuit that records the arrival time of the first photon from each laser pulse. Microlens arrays can be used to increase the optical fill factor for each APD pixel. The APD configuration is shown in Figure 11.

A Geiger-mode APD is biased beyond the impactionization avalanche breakdown voltage for the diode. The amount of bias beyond breakdown is called the over-bias. When the APD fires, a circuit element is used to drop the bias to less than breakdown voltage for some period of time ("dead" time or re-arm time), before overbias is reapplied. Although excess noise due to the presence of both conduction and valence-band multiplication in linear mode avalanche photodiodes limits their sensitivity, both electron and hole multiplication are essential for Geiger-mode breakdown in APDs. The primary metrics for G-M APD sensitivity are the photon detection efficiency and the dark count rate. Dark count rate is also sometimes specified as the probability of a dark count occurring in a single time bin (per ns, for example).

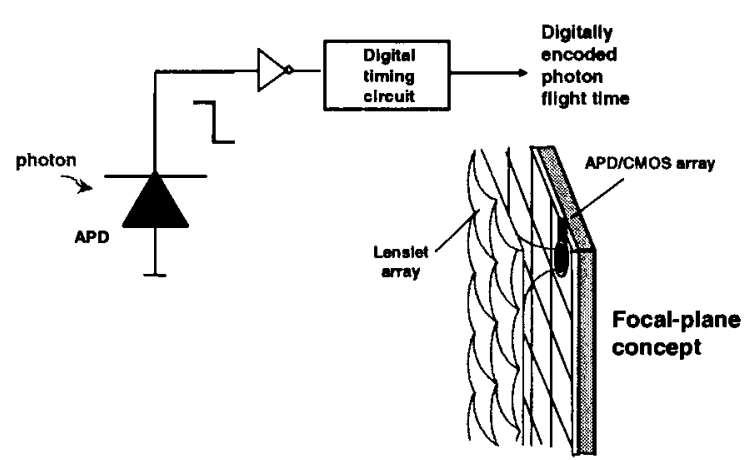

Figure 11: Geiger-Mode APD Configuration

\section{Beacon Uplink}

The laser power required for a ground-based beacon is dominated by turbulence effects. Turbulence spreads the beam, reducing mean irradiance at Mars, and causes fluctuations in the instantaneous received power.

Power requirements for a beacon that serves simply as a pointing reference for the Mars terminal are plotted in Figure 12 as a function of the aperture diameter of the beacon transmitter. The three curves in each plot represent the beacon laser powers that would be required if the beacon were completely uncompensated for atmospheric turbulence, if the turbulence-induced jitter were perfectly tracked out, and if the jitter were partially rejected, as allowed by tilt anisoplanatism. Only when the point-ahead angle is zero can the full-track curve be realized.

Further study is required to design an uplink communications function that is consistent with the use of the beacon as a pointing/tracking reference. It is expected that the sensitivity will be $1-100$ photons/bit, in which case the beacon required for a pointing reference would provide data rates from 1- 
$100 \mathrm{~kb} / \mathrm{sec}$. Even the FPA tracking a low-power beacon can provide some comm functionality, but the data rate is likely to be of order $1 \mathrm{~b} / \mathrm{s}$. Because of the widely varying beacon power that will be available from a ground-based beacon, it is desirable to design a system that can easily accommodate a wide range of data rates.

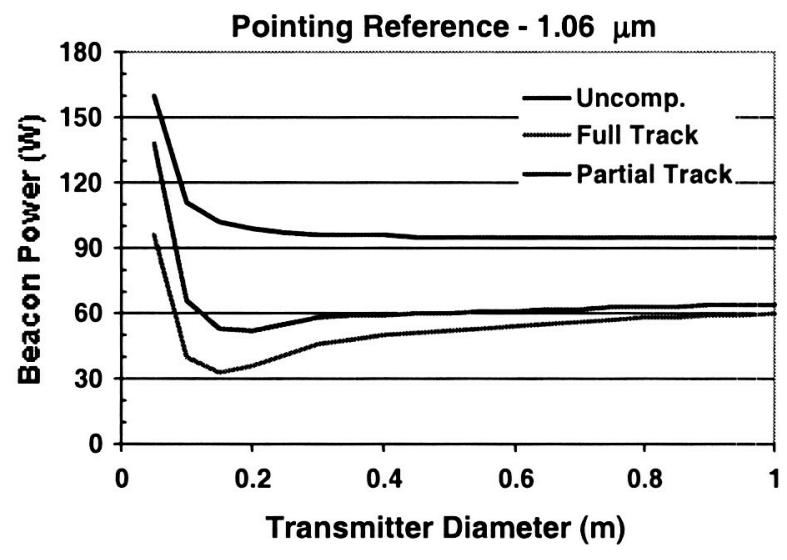

Figure 12: Beacon Power Requirements

The concept developed for implementing the beacons is to incorporate six, $30 \mathrm{~cm}$ aperture telescopes on a single equatorial mount. Optical power will be supplied by commercially available $20 \mathrm{~W}$ YDFA fiber amplifiers. Each telescope will use an amplifier for a total transmitted power of $120 \mathrm{~W}$.

The configuration of the beacon transmitter assembly is shown in Figure 13.

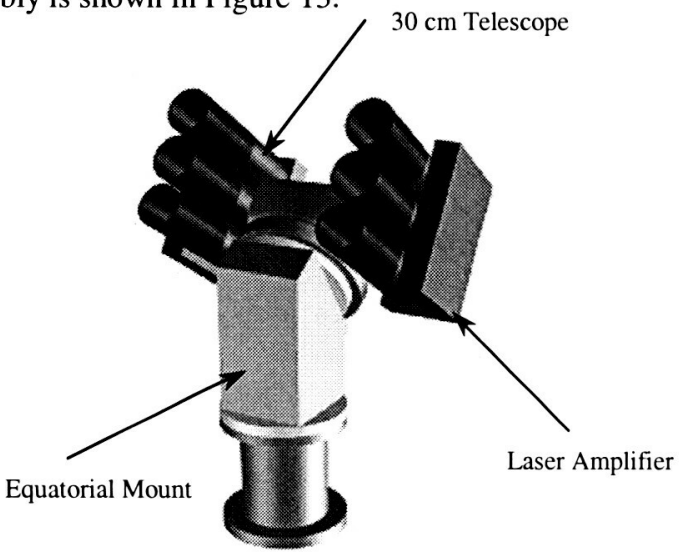

Figure 13. Beacon Transmitter Configuration

\section{DEMONSTRATION OPERATIONS}

The demonstration will last at least through one Earth year on orbit at Mars. Besides the increased data rates over today's RF systems, the demonstra- tion will address operability issues such as predefined sequence operation, link set up and tear down, and weather mitigation techniques.

The mission operations for the MTO spacecraft and the lasercom demonstration are intimately intertwined. The unique nature of the demo is that there is a path to and from MTO that is outside the usual Deep Space Network RF connection. Commands for the lasercom terminal can be sent via either the optical uplink or via the RF uplink. There are two paths for getting engineering data, again via optical or RF, and the terminal will be able to transmit "science" data. The Demonstration Operations Center (DOC) coordinates all lasercom activities and provides an interface to MTO operations.

The primary commanding mode for the lasercom terminal is via the DSN. For each lasercom pass, the desired operations (time to turn on/off, data source selection, data rate, etc.) will be predefined, sent from the DOC to MTO operations which will package them appropriately to send to the spacecraft via the DSN. Some "real-time" commanding via this link is desired to deal with weather and atmospheric conditions which may require changing, for example, the data rate. These commands, generated at the DOC, can change the internal configuration (data rate, etc.) of the lasercom terminal but will be firewalled so that no commands may be forwarded from the terminal to the spacecraft.

On the telemetry side there are again two paths, though for somewhat different reasons. Data (science or engineering telemetry) can be sent to Earth via the lasercom terminal. The selection of data, formatting, etc is done by the avionics and the lasercom accesses the appropriate space in storage to extract the data, add its own internal formatting and error-correction coding, and then transmit it to Earth. The data may be the same data that is being transmitted over the RF links, albeit potentially at different rates. It is possible that the lasercom terminal may add/multiplex additional engineering data into the data stream. The spacecraft monitors terminal parameters like power and includes those in engineering telemetry that is passed over the RF link. In addition to these, there are many 'test points" within the lasercom terminal that are available and that data can be requested and then sent via $\mathrm{RF}$ as part of the engineering telemetry. It is worth noting that, at least during the initial stages of the demonstration, a DSN pass must be scheduled to coincide with each optical pass to allow downlinking of the terminal engineering data. 
Due to the vagaries of weather and atmospheric conditions, operations strategies for mitigation of these effects will be explored. One possibility would be to have multiple terminals within the same beam simultaneously receive the same data to guarantee a reasonably high percentage of the time getting through to at least one terminal. On the other hand, buffering and retransmission strategies can be used to downlink the data to single geographically (and hopefully meteorologically) diverse stations in a form of temporal diversity.

\section{CONCLUSION}

High-rate, low mission impact communications will revolutionize deep space science. Data rates that are $10-1000$ times more capable than current RF systems will allow new kinds of connectivities and enable new kinds of scientific investigations. Except for the long inherent delays due to the vast distances involved, establishing a "virtual presence" will be possible, enabling both undreamed of planetary sensors and high-rate communications with future astronauts. The lasercom terminal to be flown on the Mars Telecom Orbiter by NASA in 2009 will be the first deep space demonstration of this revolutionary technology. The knowledge and experience gained will enable NASA to design, procure, and operate cost-effective future deep space optical communications systems.

\section{ACKNOWLEDGEMENTS}

The work described in this paper was carried out at NASA's Goddard Space Flight Center, through NASA sponsorship at Lincoln Laboratory, Massachusetts Institute of Technology, under contract with the Air Force, and at the Jet Propulsion Laboratory, California Institute of Technology, under contract with NASA.

\section{REFERENCES}

1 Michael F. Luniewicz, J. Murphy, Edward O'Neil, Dale T. Woodbury, Marcus Schulthess, "Testing the inertial pseudo-star reference unit," SPIE Proceedings Vol. 2221: Acquisition, Tracking, and Pointing VIII, ISBN: 0-81941525-1, 732 pages Published 1994, Meeting Date: 04/04 - 04/08/94, Orlando, FL, USA 